\title{
Effect of population imbalance on the Berezinskii-Kosterlitz-Thouless phase transition in a superfluid Fermi gas
}

\author{
J. Tempere, ${ }^{1,2}$ S. N. Klimin ${ }^{1, *}$ and J. T. Devreese ${ }^{1, \dagger}$ \\ ${ }^{1}$ TFVS, Universiteit Antwerpen, B-2020 Antwerpen, Belgium \\ ${ }^{2}$ Lyman Laboratory of Physics, Harvard University, Cambridge, Massachusetts 02138, USA
}

(Received 9 March 2009; published 27 May 2009)

\begin{abstract}
The Berezinskii-Kosterlitz-Thouless (BKT) mechanism describes the breakdown of superfluidity in a twodimensional Bose gas or a two-dimensional gas of paired fermions. In the latter case, a population imbalance between the two pairing partners in the Fermi mixture is known to influence pairing characteristics. Here, we investigate the effects of imbalance on the two-dimensional BKT superfluid transition and show that superfluidity is even more sensitive to imbalance than for three-dimensional systems. Finite-temperature phase diagrams are derived using the functional integral formalism in combination with a hydrodynamic action functional for the phase fluctuations. This allows to identify a phase-separation region and tricritical points due to imbalance. In contrast to superfluidity in the three-dimensional case, the effect of imbalance is also pronounced in the strong-coupling regime.
\end{abstract}

DOI: $10.1103 /$ PhysRevA.79.053637

PACS number(s): 03.75.Ss, 05.30.Fk, 03.75.Lm

\section{INTRODUCTION}

Quantum phenomena which occur at very low temperatures are a subject of intense experimental and theoretical study. Recent progress in the experimental investigation of ultracold atoms stimulated an unprecedented interest to the theoretical problems of condensation of cold bosons and pairing of interacting fermions (see, e.g., the review [1] and references therein). These phenomena are related to a variety of objects including stars, dense nuclear and quark matter, and plasma systems [2-4].

Phase transitions of quantum systems strongly depend on their dimensionality. Two-dimensional (2D) Fermi gases have remarkable features, which are not observed in three dimensions. The quasi-2D regime for cold atoms can be reached using a sufficiently strong confinement of atoms along one direction so that they occupy only the lowest size quantization subband. Advances in pairing of trapped cold atomic Fermi gases with a controlled geometry of a trapping potential allow experimentalists to realize systems of different (quasi)dimensionalities. Both condensation of bosonic atoms [5] and pairing of fermions [6] has been observed recently in optical lattices.

The Mermin-Wagner-Hohenberg theorem [7] shows that in a uniform two-dimensional (2D) system, the long-range order is destroyed by thermal fluctuations so that 2D Bose gases cannot undergo Bose-Einstein condensation at nonzero temperatures [8-10]. Nevertheless, these two-dimensional systems can form a "quasicondensate" and exhibit superfluidity [11]. Kosterlitz and Thouless [12] showed that the mechanism driving the superfluid-to-normal phase transition in this case is the proliferation of vortices and antivortices above a critical temperature $T_{B K T}$, spoiling the phase coher-

\footnotetext{
*On leave from Department of Theoretical Physics, State University of Moldova, str. A. Mateevici 60, MD-2009 Kishinev, Republic of Moldova.

${ }^{\dagger}$ Also at Technische Universiteit Eindhoven, P.O. Box 513, 5600 MB Eindhoven, The Netherlands.
}

ence. The experimental situation differs from that considered by Mermin, Wagner, and Hohenberg in that the gas is not uniform. The presence of a trapping potential in the experimental realizations changes the density of states, and in a confined 2D system, true Bose-Einstein condensation does become possible [13]. However, the Berezinskii-KosterlitzThouless (BKT) mechanism continues to play a role in the suppression of superfluidity also in trapped systems [14]. Indeed, a BKT-type crossover in a trapped quantum degenerate gas of ${ }^{87} \mathrm{Rb}$ has recently been observed by Hadzibabic et al. [15] using an interference technique to detect phase defects [16]. This has stimulated renewed theoretical interest in the BKT transition in trapped dilute gases [17].

The discussion in the above paragraph focuses on Bose gases. Rice [18] and Schmitt-Rink et al. [19] extended these results to the case of a gas of paired fermions. The pairing characteristics depend on the interatomic interaction strength. The superfluid phase transition in a 2D Fermi gas at $T=0$ has been studied by Randeria $e t$ al. [20] in a wide range of interaction strengths from the weak-coupling limit, where the Bardeen-Cooper-Schrieffer (BCS) pairing regime is realized, to the strong-coupling limit which corresponds to the Bose condensation of interacting bound pairs. Petrov et al. [21] analytically investigated pairing of a quasi 2D Fermi gas at a finite temperature in the weak-coupling BCS limit and in the strong-coupling limit where the BKT superfluid transition occurs.

The influence of spin imbalance on the superfluid properties of a Fermi gas is of particular interest. A phase separation between the superfluid and normal components of an interacting Fermi gas of cold atoms with unequal spin populations in three-dimensional (3D) was observed in Refs. [22-24]. In these experiments, both the interaction strength between ultracold fermions and the population imbalance can be controlled using the Feshbach resonance. Allowing the control of population or mass imbalance opens a unique possibility to investigate the stability of fermion pairing and, in particular, to determine experimentally the equation of state for an imbalanced fermion system [24].

The BKT phase transition for a balanced 2D Fermi gas with the $s$-wave scattering in the balanced case has been 
theoretically analyzed by Botelho and de Melo [25]. They treated the fermion pairing in $2 \mathrm{D}$ by the path integral technique taking into account phase fluctuations. Within a similar approach, using an effective Hamiltonian which involves fermions interacting with each other and with dressed molecules, the BKT transition has been considered for a quasi-2D trapped Fermi gas [26].

In the present work, we extend the approach of Ref. [25] to investigate the effect of population imbalance on the BKT phase transition in a 2D Fermi gas. Using the HubbardStratonovich transformation, we derive a hydrodynamic effective bosonic action, which in the limiting case of a balanced gas is reduced to the effective action of Ref. [25]. On the basis of the obtained effective action, we analyze phase diagrams for an interacting, imbalanced Fermi gas in 2D. The paper is organized as follows. In Sec. II, we describe the theoretical formalism for interacting fermions in 2D. In Sec. III, we analyze the dependence of the critical temperature of the BKT transition on the coupling strength and on the population imbalance. The section is followed by conclusions (Sec. IV).

\section{FUNCTIONAL INTEGRAL DESCRIPTION}

\section{A. General formalism}

We consider a two-component gas of interacting fermions in 2D, with the $s$-wave pairing and with a population imbalance. The partition function of the system of fermions in $2 \mathrm{D}$ is expressed as the path integral over Grassmann variables $\left[\bar{\psi}_{\mathbf{x}, \tau, \sigma}, \psi \mathbf{x}, \tau, \sigma\right]$,

$$
\mathcal{Z} \propto \int \mathcal{D}\left[\bar{\psi}_{\mathbf{x}, \sigma}(\tau), \psi_{\mathbf{x}, \sigma}(\tau)\right] \exp (-S) .
$$

The action functional

$$
S=S_{0}+S_{\text {int }}
$$

is a sum of the free-fermion and interaction contributions,

$$
S_{0}=\int_{0}^{\beta} d \tau \int d^{2} \mathbf{x} \sum_{\sigma=\uparrow, \downarrow}\left[\bar{\psi}_{\mathbf{x}, \sigma}(\tau)\left(\frac{\partial}{\partial \tau}-\nabla_{\mathbf{x}}^{2}-\mu_{\sigma}\right) \psi_{\mathbf{x}, \sigma}(\tau)\right],
$$

$$
S_{\text {int }}=\int_{0}^{\beta} d \tau \int d^{2} \mathbf{x} \int d^{2} \mathbf{y} V(\mathbf{x}-\mathbf{y}) \bar{\psi}_{\mathbf{x}, \uparrow}(\tau) \bar{\psi}_{\mathbf{y}, \downarrow}(\tau) \psi_{\mathbf{y}, \downarrow}(\tau) \psi_{\mathbf{x}, \uparrow}(\tau),
$$

where $\beta=\frac{1}{k_{B} T}$ is the inverse to the temperature. We use the units in which $\hbar=1$, the fermion mass $m=1 / 2$, and the Fermi energy $E_{F} \equiv\left(2 \pi n_{0}\right)^{2 / 3} /(2 m)=1$ (where $n_{0}$ is the fermion density in 2D). We express the results below in terms of the averaged chemical potential $\mu=\left(\mu_{\uparrow}+\mu_{\downarrow}\right) / 2$ determining the total number of fermions and the imbalance potential $\zeta$ $=\left(\mu_{\uparrow}-\mu_{\downarrow}\right) / 2$. For the interaction potential, we use the separable expression proposed in Refs. [25,27],

$$
V_{\mathbf{k}, \mathbf{k}^{\prime}}=g \Gamma_{\mathbf{k}} \Gamma_{\mathbf{k}^{\prime}},
$$

where $g$ is the interaction strength. The factor $\Gamma_{\mathbf{k}}$ describes a finite-range potential,

$$
\Gamma_{\mathbf{k}}=\left(1+\frac{k}{k_{0}}\right)^{-1 / 2}
$$

where $R \sim k_{0}^{-1}$ plays the role of the interaction range. The particular case of the contact interaction corresponds to $k_{0}$ $\rightarrow \infty$ so that $\Gamma_{\mathbf{k}} \rightarrow 1$. The interaction term $S_{\text {int }}$ of the action functional is then given by

$$
S_{i n t}=\int_{0}^{\beta} d \tau \frac{g}{L^{2}} \sum_{\mathbf{q}} \bar{B}_{\mathbf{q}}(\tau) B_{\mathbf{q}}(\tau),
$$

where $L$ is the lateral size of the $2 \mathrm{D}$ system, and the collective coordinates $B_{\mathbf{q}}(\tau)$ are determined as

$$
B_{\mathbf{q}}(\tau) \equiv \sum_{\mathbf{k}} \Gamma_{\mathbf{k}} a_{-\mathbf{k}+\mathbf{q} / 2, \downarrow}(\tau) a_{\mathbf{k}+\mathbf{q} / 2, \uparrow}(\tau) .
$$

Further on, we apply the Hubbard-Stratonovich (HS) transformation. Introducing the extended action

$$
S_{\text {ext }}=S-\int_{0}^{\beta} d \tau \frac{1}{g} \sum_{\mathbf{q}} \bar{\phi}_{\mathbf{q}}(\tau) \phi_{\mathbf{q}}(\tau),
$$

with the auxiliary Bose field (HS field) $\phi_{\mathbf{q}}(\tau)$ and performing the shift of boson coordinates, which eliminates the fermionfermion interaction term $S_{i n t}$, the HS transformation results in the action

$$
\begin{aligned}
S_{\text {ext }}= & S_{0}-\int_{0}^{\beta} d \tau \frac{1}{L} \sum_{\mathbf{q}}\left[\bar{B}_{\mathbf{q}}(\tau) \phi_{\mathbf{q}}(\tau)+B_{\mathbf{q}}(\tau) \bar{\phi}_{\mathbf{q}}(\tau)\right] \\
& -\int_{0}^{\beta} d \tau \frac{1}{g} \sum_{\mathbf{q}} \bar{\phi}_{\mathbf{q}}(\tau) \phi_{\mathbf{q}}(\tau) .
\end{aligned}
$$

Because the phase fluctuations about the saddle point are, in general, not small, the boson (HS) and fermion variables in the coordinate representation are transformed as [28]

$$
\phi_{\mathbf{r}}(\tau)=\phi_{\mathbf{r}}^{\prime}(\tau) e^{i \theta_{\mathbf{r}}(\tau)}, \quad \psi_{\mathbf{r}, \sigma}(\tau)=\psi_{\mathbf{r}, \sigma}^{\prime}(\tau) e^{i \theta_{\mathbf{r}}(\tau) / 2} .
$$

In the same formalism for a Fermi gas in $3 \mathrm{D}$, the further step is the path integration over fermion variables and the expansion of the resulting bosonic action over fluctuations about the saddle point [29]. This method provides a description of a superfluid phase transition between the normal phase and the true condensate of fermion pairs. In the 2D case, at least when restricting the expansion by quadratic fluctuations, the superfluidity occurs only at $T=0$ [19]. Traven [30] showed that the interaction between fluctuations of the pairing field in a 2D attractive Fermi gas allows a superfluid phase transition at a very low temperature. However, the superfluid state can exist in a 2D Fermi gas at relatively high temperatures as a quasicondensate, i.e., a state with fermion pairing "where the density fluctuations are suppressed but the phase still fluctuates" [31]. In two dimensions, the quasicondensate can be realized through bound vortex-antivortex pairs [1].

\section{B. Fluctuating phase}

After integrating out the fermion (Grassmann) variables, an effective action in the Bose field $\phi_{\mathbf{r}}$ is obtained. The re- 
maining functional integral over this Bose field cannot be taken in general. Several levels of approximation can be made to get results. The crudest approximation is the meanfield approximation which replaces the field by a constant, $\phi_{\mathbf{r}} \rightarrow|\Delta|$, the saddle point. To improve on this, fluctuations around the saddle point can still be taken into account; this can be done in an exact way only up to quadratic order in the fluctuation. One can choose to write the fluctuations as $\phi_{\mathbf{r}}$ $\rightarrow|\Delta|+\delta_{\mathbf{r}}$, with $\delta_{\mathbf{r}}$ being complex; this corresponds to the NSR [29] approach that investigates the presence of a real condensate rather than a quasicondensate. Alternatively the NSR fluctuations can be written as amplitude and phase fluctuations, $\phi_{\mathbf{r}} \rightarrow\left(|\Delta|+\left|\delta_{\mathbf{r}}\right|\right) e^{i \theta_{\mathbf{r}}}$, where $\left|\delta_{\mathbf{r}}\right|$ and $\theta_{\mathbf{r}}$ are the real fields. In order to treat the quasicondensate state, relevant for the 2D case, we have to focus on phase fluctuations: that corresponds to setting $\phi_{\mathbf{r}} \rightarrow|\Delta| e^{i \theta_{\mathbf{r}}}$. We will simplify the notation and write $|\Delta|=\Delta$ for the energy gap parameter of the Bogoliubov excitations. Moreover, we will assume that the remaining fluctuation field $\theta_{\mathbf{r}}$ varies slowly as a function of position and time with respect to the variations in the fermion fields. A similar assumption was used in Refs. [26,28]. The resulting hydrodynamic action is structurally similar to the saddle-point action for imbalanced fermions in $2 \mathrm{D}$ [32]

$$
\begin{aligned}
S_{e f f}= & -\int_{0}^{\beta} d \tau \int d^{2} \mathbf{r} \frac{1}{L^{2}} \sum_{\mathbf{k}}\left[\frac { 1 } { \beta } \operatorname { l n } \left(2 \cosh \beta \zeta_{\mathbf{k}}\right.\right. \\
& \left.\left.+2 \cosh \beta \widetilde{E}_{\mathbf{k}}\right)-\widetilde{\xi}_{\mathbf{k}}\right]-\frac{\beta L^{2}}{g} \Delta^{2},
\end{aligned}
$$

in which the fermion energy $\xi_{\mathrm{k}}=k^{2}-\mu$, the Bogoliubov excitation energy $E_{\mathbf{k}}=\sqrt{\xi_{\mathbf{k}}^{2}+\Delta^{2} \Gamma_{\mathbf{k}}^{2}}$ and the imbalance potential $\zeta$ are replaced by expressions depending on boson coordinates:

$$
\begin{gathered}
\tilde{\xi}_{\mathbf{k}}=k^{2}-\tilde{\mu}, \quad \tilde{E}_{\mathbf{k}}=\sqrt{\tilde{\xi}_{\mathbf{k}}^{2}+\Delta^{2} \Gamma_{\mathbf{k}}^{2}}, \\
\tilde{\mu}=\mu-\frac{i}{2} \frac{\partial \theta}{\partial \tau}-\frac{1}{4}(\nabla \theta)^{2}+\frac{i}{2} \nabla^{2} \theta, \\
\zeta_{\mathbf{k}}=\zeta-\nabla \theta \cdot \mathbf{k} .
\end{gathered}
$$

Keeping the quadratic-order fluctuation terms we arrive at the action $S_{e f f}$ as the sum of the saddle-point action $S_{s p}$, which coincides with expression (5) of Ref. [32], and the diagonal quadratic form of the phase fluctuations

$$
S_{f l}=\frac{1}{2} \int_{0}^{\beta} d \tau \int d^{2} \mathbf{r}\left[A\left(\frac{\partial \theta}{\partial \tau}\right)^{2}+\rho_{s}(\boldsymbol{\nabla} \theta)^{2}\right] .
$$

The coefficients in the fluctuation action are the pair superfluid density

$$
\rho_{s}=\frac{1}{L^{2}} \sum_{\mathbf{k}}\left[\frac{1}{2}\left(1-\frac{\xi_{k}}{E_{k}} X_{k}\right)-\frac{k^{2}}{2} Y_{\mathbf{k}}\right]
$$

and the constant

$$
A=\frac{1}{4 L^{2}} \sum_{\mathbf{k}}\left(\frac{\Gamma_{k}^{2} \Delta^{2}}{E_{\mathbf{k}}^{3}} X_{k}+\frac{\xi_{k}^{2}}{E_{k}^{2}} Y_{k}\right)
$$

where $X_{k}$ is the function

$$
X_{k}=\frac{\sinh \beta E_{k}}{\cosh \beta \zeta+\cosh \beta E_{k}}
$$

and $Y_{k}$ is the extension of the Yoshida distribution to imbalanced fermions:

$$
Y_{k}=\beta \frac{\cosh \beta \zeta \cosh \beta E_{k}+1}{\left(\cosh \beta \zeta+\cosh \beta E_{k}\right)^{2}} .
$$

Note that expression (16) for $\rho_{s}$ agrees with the definition of superfluid density used for a 3D balanced Fermi gas [33].

The action in Eq. (15) describes a Bose gas of spin waves [25] with the energy spectrum $\omega_{k}$ given by

$$
\omega_{k}=c k, \quad c=\sqrt{\frac{\rho_{s}}{A}} .
$$

The spin-wave contribution to the thermodynamic potential is given by the expression

$$
\Omega_{s w}=\frac{1}{\beta} \sum_{\mathbf{k}} \ln \left(1-e^{-\beta \omega_{k}}\right) .
$$

The total thermodynamic potential of the fermion system taking into account phase fluctuations is the sum of the spinwave term [Eq. (19)] and the saddle-point contribution, which is provided by the saddle-point action,

$$
\Omega_{s p}=\sum_{\mathbf{k}}\left[\frac{1}{\beta} \ln \left(2 \cosh \beta \zeta+2 \cosh \beta E_{\mathbf{k}}\right)-\xi_{\mathbf{k}}\right]-\frac{\beta L^{2}}{g} \Delta^{2} .
$$

For an imbalanced quasi-2D Fermi gas in an optical potential, the mean-field zero-temperature phase diagrams were analyzed in Ref. [32] on the basis of this saddle-point action (neglecting spin-wave contributions).

The gap parameter $\Delta$ for an imbalanced Fermi gas is determined through the minimization of the saddle-point thermodynamic potential $\Omega_{s p}$ as a function of $\Delta$ at given $\beta, \mu, \zeta$ :

$$
\left(\frac{\partial \Omega_{s p}}{\partial \Delta}\right)_{\beta, \mu, \Delta}=0 .
$$

For a complete determination of thermodynamic parameters at a given temperature, the minimum condition [Eq. (21)] is solved jointly with the number equations:

$$
n \equiv-\left(\frac{\partial}{\partial \mu} \frac{\Omega}{L^{2}}\right)_{\beta, \zeta, \Delta}=\frac{1}{2 \pi}, \quad \delta n \equiv-\left(\frac{\partial}{\partial \zeta} \frac{\Omega}{L^{2}}\right)_{\beta, \mu, \Delta}=\frac{1}{2 \pi} \frac{\delta n}{n},
$$

where $n$ and $\delta n$ are the total fermion density $n$ and the density difference $\delta n$, respectively.

\section{BKT transition temperature}

The coupled gap and number Eqs. (21) and (22) have to be solved together. In Eq. (22), three different levels of approximation can be made, in analogy to the approximations for the Bose field $\phi_{\mathbf{r}}(\tau)$ as discussed in the beginning of the previous subsection. 
The first and simplest case is the mean-field approximation (as described in Refs. [25,26]), where we use $\Omega=\Omega_{s p}$. This corresponds to setting the (Hubbard-Stratonovic) Bose field equal to a constant $\phi=\Delta_{M F}$ (both constant in amplitude and in phase). The constant can be determined by minimizing $\Omega_{s p}$ and allows to determine the phase-transition line between the normal state, in which $\Delta_{M F}=0$, and the quasicondensate of fermion pairs, in which $\Delta_{M F} \neq 0$. The temperature separating the aforementioned phases will be denoted by $T_{M F}$. For a balanced gas, the phase transition between normal and paired states is of the second order. For an imbalanced gas, also the first-order phase transition between the normal and paired states is possible.

The paired state below $T_{M F}$ is not always superfluid. Proliferating vortices and antivortices destroys phase coherence in the quasicondensate and suppresses superfluidity [12]. The mean-field approximation does not allow us to determine the temperature $T_{B K T}$ separating the superfluid quasicondensate state from the nonsuperfluid paired state. To investigate the question of superfluidity and determine the BKT temperature, we need to use $\Omega=\Omega_{s p}+\Omega_{s w}$ in Eq. (22), where $\Omega_{s w}$ is the free energy contribution [Eq. (19)] from the phase fluctuations. This corresponds to giving the (HubbardStratonovic) Bose field a constant amplitude, but allowing its phase to fluctuate freely. The BKT transition temperature is then a root of the universal Nelson-Kosterlitz equation [34]

$$
T_{B K T}-\frac{\pi}{2} \rho_{s}\left(T_{B K T}\right)=0 .
$$

As distinct from the aforesaid phase transition at $T=T_{M F}$, the BKT phase transition at $T=T_{B K T}$ is characterized by an abrupt change in the superfluid density from zero to a finite value satisfying Eq. (16). The phase-fluctuation contribution to the density vanishes at the phase boundary between the paired state and the normal state because at $\Delta_{M F}=0$ the superfluid density $\rho_{s}$ turns to zero.

Finally, the approximation of setting $\Omega=\Omega_{s p}+\Omega_{s w}$ in Eq. (22) does not allow us to determine whether the superfluid quasicondensate can form a true condensate below some critical temperature $T_{B E C}$. Note that the emergence of a true condensate in $2 \mathrm{D}$ is only possible in a finite system, as $T_{B E C} \rightarrow 0$ for $L \rightarrow \infty$. To determine the critical temperature we should use $\Omega=\Omega_{s p}+\Omega_{\text {flucts }}$ in Eq. (22), where $\Omega_{\text {flucts }}$ contains contributions from both amplitude and phase fluctuations in the Hubbard-Stratonovic Bose field.

\section{RESULTS AND DISCUSSION}

When $T_{M F}>0$, Eq. (23) can be satisfied if the superfluid density $\rho_{s} \neq 0$. At $T=T_{M F}$, the superfluid density for a balanced Fermi gas turns to zero. Therefore for a balanced Fermi gas $T_{B K T}$ is always lower than $T_{M F}$. On the contrary, in the case of unequal "spin-up" and "spin-down" populations, for a sufficiently high population imbalance the phase transition at $T=T_{M F}$ can be of the first order when the gap parameter $\Delta$ changes discontinuously from zero to a finite value. In this case, as shown below, $T_{B K T}$ and $T_{M F}$ can coincide in a definite range of the coupling strength.
In the region between $T_{B K T}$ and $T_{M F}$, the superfluidity is destroyed owing to a proliferation of free vortices. However, the phase of a $2 \mathrm{D}$ Fermi gas in this region is not a uniform normal state because the gap parameter for $T_{B K T}<T<T_{M F}$ can be other than zero so that fermion pairing can occur in that temperature region. The region between $T_{B K T}$ and $T_{c}$ can be therefore attributed to a state in which pairing can occur but the phase coherence is destroyed. For slightly higher temperatures with respect to $T_{B K T}$, vortices form a disordered gas of phase defects [1]. For higher temperatures the concept of vortices is inapplicable due to density fluctuations.

In general, there are the following regions in phase diagrams for an imbalanced Fermi gas in 2D: (1) the normal state in which the gap parameter $\Delta=0$, (2) the superfluid quasicondensate state in which $\Delta \neq 0$ and $\rho_{s} \neq 0$, (3) the state in which pairing is possible but without phase coherence, and (4) the phase-separation region, which appears due to a population imbalance. In the latter one, no solution exist for the set of Eqs. (21) and (22). Therefore, in the phaseseparation region the system will unmix in a phase with lower (or no) imbalance and the normal phase at higher imbalance. At the first-order phase transition, the system abruptly passes through the phase-separation region.

In Fig. 1, we represent phase diagrams in the variables $\left\{T, E_{b}\right\}$, where $T$ is the temperature and $E_{b}$ is the binding energy of a two-particle bound state in $2 \mathrm{D}$. The phase diagrams in Fig. 1 are obtained at a given value of the imbalance chemical potential $\zeta$. The top panel shows the balanced case $(\zeta=0)$, and the middle and lower panels illustrate how the diagram changes with increasing difference between the chemical potentials of up and down species. The energy $E_{b}$ is the parameter which characterizes the coupling strength of the fermion-fermion attractive interaction. In two dimensions, the strength $g$ of the contact interaction is related to this binding energy through [20]

$$
\frac{1}{g}=\frac{m}{4 \hbar^{2}}\left[i-\frac{\ln \left(E / E_{b}\right)}{\pi}\right] .
$$

The binding energy itself can be related to the experimental parameters. The two-dimensional system is created through a strong confinement of the third direction; in general this strong confinement can be associated with a harmonic potential with frequency $\omega_{L}$ (and oscillator length $\ell_{L}$ ). The twoparticle bound state exists for all values of the (3D) $s$-wave scattering length $a_{s}$ of the fermionic atoms and its binding energy is given by

$$
E_{b}=\frac{C \hbar \omega_{L}}{\pi} \exp \left(\sqrt{2 \pi} \frac{\ell_{L}}{a_{s}}\right),
$$

with $C \approx 0.915$ (cf. Ref. [35]).

The dashed curves in Fig. 1 correspond to the phasetransition curves, $T_{M F}\left(E_{b}\right)$, within the mean-field approach. When the system is imbalanced, two changes occur with respect to the balanced case: (i) below a certain value of the binding energy, $T_{M F} \rightarrow 0$, and (ii) a tricritical point appears on this curve. The mean-field phase-transition curve splits below this tricritical point and the phase-separation region opens up. In this tricritical point, there is coexistence of the 

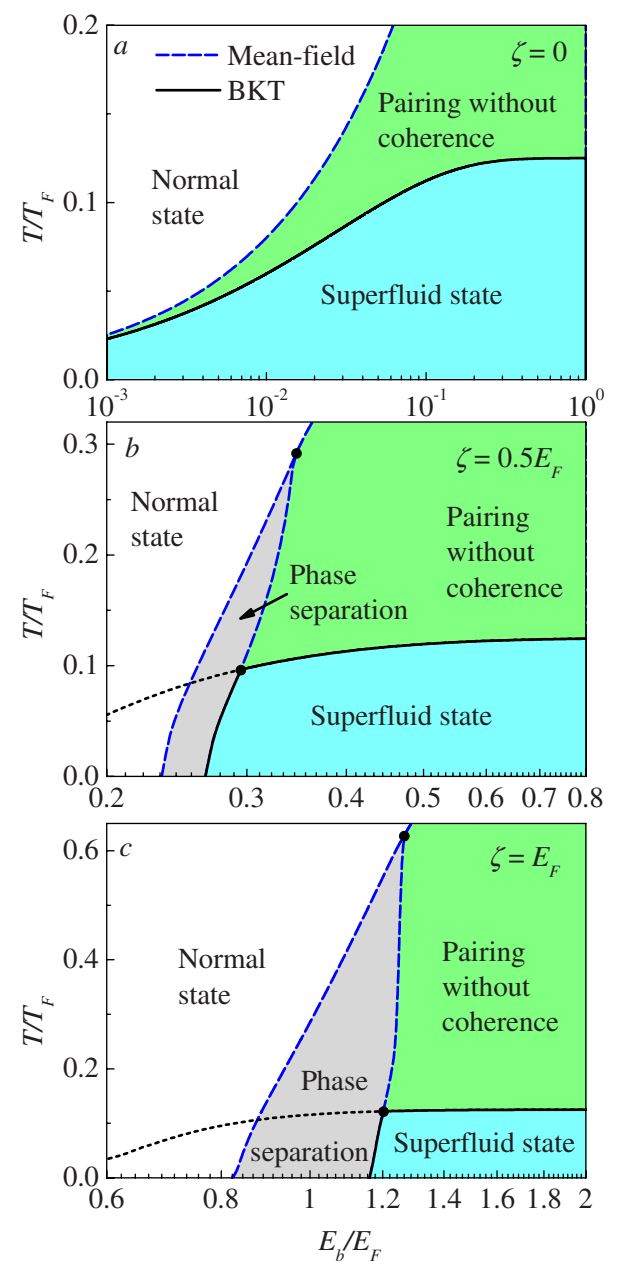

FIG. 1. (Color online) Phase diagrams for a 2D imbalanced Fermi gas in the variables $\left(T, E_{b}\right)$ for different values of the imbalance potential $\zeta$. The dotted curve indicates the BKT transition to a metastable superfluid state.

paired state without phase coherence, the normal state, and the phase-separation region. The area of the phase-separation region is narrow and broadens with increasing the imbalance potential.

The full curve corresponds to the BKT phase-transition line $T_{B K T}\left(E_{b}\right)$. For the balanced case, we retrieve the results obtained in Ref. [25] and find that the BKT transition temperature is nonzero for any finite binding energy. In particular, we find that in the strong-coupling limit (for large $E_{b}$ ), the superfluid density $\rho_{s}$ rises to its maximum, equal to half the total fermion density, so that the BKT transition temperature obtained from Eq. (23) tends to a finite value $T_{B K T}^{(\max )}$ $=(1 / 8) T_{F}$. When imbalance is introduced, two qualitative changes occur in the BKT transition curve. First, below a critical value of the binding energy, $T_{B K T} \rightarrow 0$. Imbalance suppresses BKT-superfluidity as it does for 3D superfluidity. The critical binding energy needed to restore superfluidity shifts to higher values as the imbalance potential grows. Second, there appears a tricritical point also on the BKT curve, indicating coexistence of the superfluid state, the state with pairing but no phase coherence, and the phase-separation region.

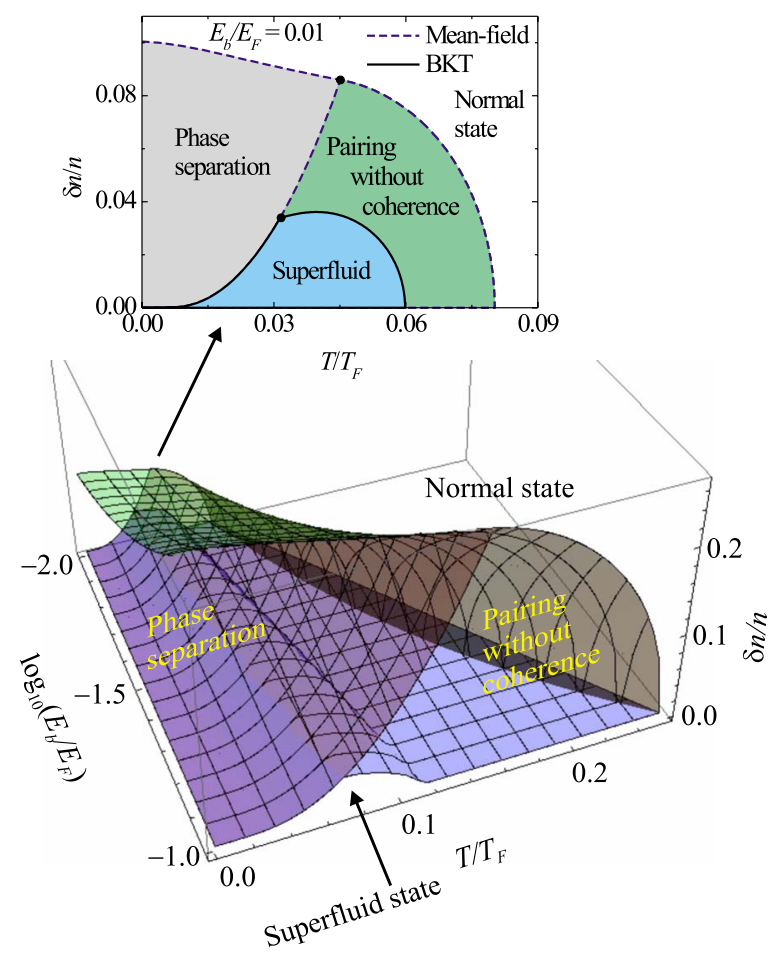

FIG. 2. (Color online) Three-dimensional phase diagram for a $2 \mathrm{D}$ imbalanced Fermi gas in the variables $\left(E_{b}, T, \frac{\delta n}{n}\right)$. The slice at $E_{b}=0.01$ shows the phase diagram in the variables $\left(T, \frac{\delta n}{n}\right)$ with the same denotations as in Fig. 1.

The dotted curve in the region of the normal state separates two regimes: (i) the regime where the thermodynamic potential contains only one minimum at $\Delta=0$ above the dotted curve and (ii) the regime where the thermodynamic potential contains two minima: the lower minimum at $\Delta=0$ and a higher one at a value $\Delta \neq 0$. This second minimum suggests the existence of a metastable superfluid state.

In Fig. 2, we choose to fix $\delta n / n$, the relative population imbalance itself, rather than the imbalance potential $\zeta$, and study the phase diagram in the variables $\left\{E_{b}, T, \frac{\delta n}{n}\right\}$. The inset shows a slice of this phase diagram at $E_{b}=0.01$. At this binding energy, the BKT transition temperature is a non-singlevalued function of $\delta n / n$. This result is not unexpected, because a similar non-single-valued behavior of the critical temperature exists also for the superfluid phase transition in a 3D Fermi gas [36]. In the 3D case this can be related to the Sarma mechanism [37]: at nonzero temperatures, a balanced superfluid system coexists with an imbalanced gas of thermal excitations-these excitations carry some of the excess majority component of the imbalanced gas.

The BKT phase transition for a Fermi gas in 2D is suppressed at lower values of the population imbalance than the superfluid phase transition for a Fermi gas in 3D. Furthermore, this suppression is more strongly revealed at higher binding energies. The reason of such a dependence of the BKT phase transition as a function of the binding energy consists in the following. Let us consider first a part of the phase diagram in the variables $\left(E_{b}, T, \frac{\delta n}{n}\right)$ taking into attention only the mean-field phase transition. Similar phase diagrams were calculated for a 3D Fermi gas in Ref. [36]. An 


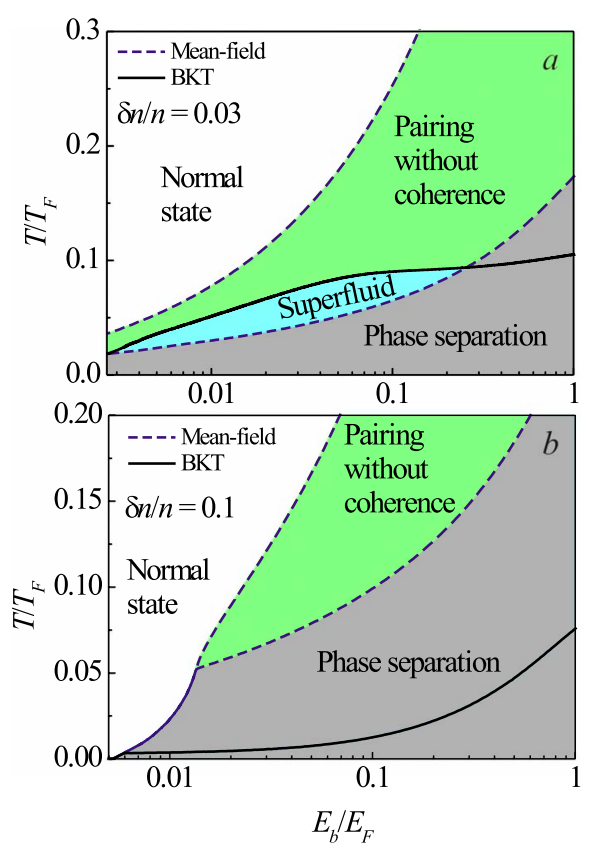

FIG. 3. (Color online) Phase diagram for an imbalanced 2D gas of cold fermions in the variables $\left(T, E_{b}\right)$ at the relative population imbalance (a) $\frac{\delta n}{n}=0.03$ and (b) $\frac{\delta n}{n}=0.1$. The denotations are the same as in Fig. 1.

important feature of phase diagrams for both $3 \mathrm{D}$ and $2 \mathrm{D}$ imbalanced Fermi gases is the fact that the phase-separation region lies at lower temperatures with respect to the superfluid state. This means that, when the temperature is gradually lowered at a fixed imbalance $\delta n / n$ (which is assumed to be sufficiently low to pass the region of the superfluid state), we can observe the superfluid phase transition from the normal state to the superfluid state, and then, when the temperature is lowered further, the superfluid state becomes nonstable in the phase-separation region. So, a sufficient lowering of the temperature leads to an instability of the superfluid state of an imbalanced Fermi gas. On the one hand, with increasing the coupling strength, the phaseseparation region broadens, and its upper-temperature boundary shifts to higher temperatures. On the other hand, the BKT transition temperature does not unrestrictedly increase with an increasing coupling strength. As follows from the Nelson-Kosterlitz Eq. (23), the upper bound for $T_{B K T}$ is $T_{B K T}=(1 / 8) T_{F}$. This is the case when the superfluid pair density achieves its maximal possible value for a balanced gas $\rho_{s}^{(\max )}=\frac{1}{2} n=\frac{1}{4 \pi}$. As a result, with an increasing coupling strength, we arrive at a stronger overlap between the area of the superfluid quasicondensate state (below $T_{B K T}$ ) and the phase-separation region. It is natural to expect that at sufficiently high binding energy, the phase-separation region can completely cover the area below $T_{B K T}$ so that the superfluid state vanishes at all temperatures. In this connection, the study of phase diagrams for a 2D Fermi gas in the variables $\left(T, E_{b}\right)$ at a given relative population imbalance (i.e., slices of the above considered three-dimensional phase diagram at fixed $\frac{\delta n}{n}$ ) represents a particular interest.

In Fig. 3, we have plotted phase diagrams for a 2D Fermi gas in the variables $\left(T, E_{b}\right)$ at the relative population imbal- ance $\frac{\delta n}{n}=0.03$ and 0.1 . We see that even at a low population imbalance $\frac{\delta n}{n}=0.03$, the region where the superfluid state exists is relatively narrow. Furthermore, with an increasing binding energy $E_{b}$, the upper-temperature bound of the phase-separation region rises faster than the BKT transition temperature. As a result, the range of coupling strengths at which the BKT superfluid phase transition can occur is restricted both from lower and higher couplings. For the comparison, the regular superfluid phase transition in a $3 \mathrm{D}$ imbalanced Fermi gas is restricted only from lower couplings [38]. We can see from Fig. 3(b) that for a higher (but relatively low) imbalance $\frac{\delta n}{n}=0.1$, the higher-temperature bound of the phase-separation region lies higher than $T_{B K T}$ for all considered values of the binding energy $E_{b}$. As a result, for $\frac{\delta n}{n}=0.1$ the superfluid state is absent. The obtained behavior of phase diagrams for a 2D Fermi gas confirms our suggestions above.

\section{CONCLUSIONS}

In summary, we have described the effects of imbalance on the Berezinskii-Kosterlitz-Thouless superfluid transition in a 2D Fermi gas through the functional integral formalism. Owing to a population imbalance, the superfluid state cannot exist for all values of the coupling strength, but only above a certain critical binding energy which depends on the imbalance. The larger the imbalance potential, the higher is this critical binding energy. As distinct from the balanced case, there is a phase-separated region in phase diagrams, in which no uniform state can exist. As a result, tricritical points appear at the phase diagrams, in which three different regimes coexist. The area of the phase-separated region increases with increasing the population imbalance. Due to the rise of the upper-temperature bound of the instability region, the area of the superfluid state at a fixed relative population imbalance decreases with increasing the binding energy. Therefore a population imbalance is a factor destroying superfluidity in 2D systems, especially at high binding energies. The BKT transition can be experimentally observable for an imbalanced Fermi gas through the phase separation in a quasi-2D trap. As follows from the obtained results, the parameters of the state of that system (e.g., critical temperatures and/or density profiles) are expected to be substantially more sensitive to the population imbalance than the corresponding parameters for a 3D gas.

\section{ACKNOWLEDGMENTS}

The authors would like to acknowledge fruitful discussions with M. Wouters, M. Oberthaler, and H. T. C. Stoof. This work was supported by FWO-V under Projects No. G.0115.06, No. G.0356.06, No. G.0180.09N, No. G.0370.09N, the WOG under Grant No. WO.033.09N (Belgium), and INTAS under Project No. 05-104-7656. J.T. gratefully acknowledges support of the Special Research Fund of the University of Antwerp under Grant No. BOF NOI UA 2004. 
[1] I. Bloch, J. Dalibard, and W. Zwerger, Rev. Mod. Phys. 80, 885 (2008).

[2] R. Casalbuoni and G. Nardulli, Rev. Mod. Phys. 76, 263 (2004); Yu. A. Litvinov et al., Phys. Rev. Lett. 95, 042501 (2005); E. Braaten and H.-W. Hammer, Phys. Rep. 428, 259 (2006); G. Labeyrie, F. Michaud, and R. Kaiser, Phys. Rev. Lett. 96, 023003 (2006).

[3] P. F. Bedaque, Nucl. Phys. A. 697, 569 (2002); M. G. Alford, J. A. Bowers, and K. Rajagopal, Phys. Rev. D 63, 074016 (2001).

[4] A. Sedrakian and U. Lombardo, Phys. Rev. Lett. 84, 602 (2000).

[5] M. Greiner, M. O. Mandel, T. Esslinger, T. Hänsch, and I. Bloch, Nature (London) 415, 39 (2002).

[6] J. K. Chin, D. E. Miller, Y. Liu, C. Stan, W. Setiawan, C. Sanner, K. Xu, and W. Ketterle, Nature (London) 443, 961 (2006).

[7] N. D. Mermin and H. Wagner, Phys. Rev. Lett. 17, 1133 (1966); P. C. Hohenberg, Phys. Rev. 158, 383 (1967).

[8] N. N. Bogoliubov, Lectures on Quantum Statistics (Gordon and Breach, New York, 1970), Vol. 2.

[9] E. M. Lifshitz and L. P. Pitaevskii, Statistical Physics (Pergamon Press, Oxford, 1980), Pt. 2.

[10] V. N. Popov, Functional Integrals in Quantum Field Theory and Statistical Physics (Reidel, Dordrecht, 1983).

[11] V. L. Berezinskii, Sov. Phys. JETP 32, 493 (1971).

[12] J. M. Kosterlitz and D. J. Thouless, J. Phys. C 6, 1181 (1973); J. M. Kosterlitz, ibid. 7, 1046 (1974).

[13] D. S. Petrov, M. Holzmann, and G. V. Shlyapnikov, Phys. Rev. Lett. 84, 2551 (2000); V. Bagnato and D. Kleppner, Phys. Rev. A 44, 7439 (1991); J. Tempere and J. T. Devreese, Solid State Commun. 101, 657 (1997); J. P. Fernandez and W. J. Mullin, J. Low Temp. Phys. 128, 233 (2002).

[14] N. Prokof'ev and B. Svistunov, Phys. Rev. A 66, 043608 (2002); S. Sachdev and E. Demler, Phys. Rev. B 69, 144504 (2004).

[15] Z. Hadzibabic, P. Krüger, M. Cheneau, B. Battelier, and J. Dalibard, Nature (London) 441, 1118 (2006); P. Krüger, Z. Hadzibabic, and J. Dalibard, Phys. Rev. Lett. 99, 040402 (2007).

[16] Z. Hadzibabic, S. Stock, B. Battelier, V. Bretin, and J. Dalibard, Phys. Rev. Lett. 93, 180403 (2004); S. Stock, Z. Hadzibabic, B. Battelier, M. Cheneau, and J. Dalibard, ibid. 95, 190403 (2005).

[17] M. Holzmann, G. Baym, J.-P. Blaizot, and F. Laloë, Proc. Natl. Acad. Sci. U.S.A. 104, 1476 (2007); Z. Hadzibabic, P. Krüger, M. Cheneau, S. P. Rath, and J. Dalibard, New J. Phys. 10, 045006 (2008); T. P. Simula, M. J. Davis, and P. B. Blakie,
Phys. Rev. A 77, 023618 (2008); M. Holzmann and W. Krauth, Phys. Rev. Lett. 100, 190402 (2008); R. N. Bisset, M. J. Davis, T. P. Simula, and P. B. Blakie, Phys. Rev. A 79, 033626 (2009).

[18] T. M. Rice, Phys. Rev. 140, A1889 (1965).

[19] S. Schmitt-Rink, C. M. Varma, and A. E. Ruckenstein, Phys. Rev. Lett. 63, 445 (1989).

[20] M. Randeria, J. M. Duan, and L. Y. Shieh, Phys. Rev. B 41, 327 (1990).

[21] D. S. Petrov, M. A. Baranov, and G. V. Shlyapnikov, Phys. Rev. A 67, 031601(R) (2003).

[22] M. W. Zwierlein, A. Schirotzek, C. H. Schunck, and W. Ketterle, Science 311, 492 (2006); M. W. Zwierlein, C. H. Schunck, A. Schirotzek, and W. Ketterle, Nature (London) 442, 54 (2006).

[23] G. B. Partridge, W. Li, R. I. Kamar, Y.-A. Liao, and R. G. Hulet, Science 311, 503 (2006).

[24] Y. Shin, C. H. Schunck, A. Schirotzek, and W. Ketterle, Nature (London) 451, 689 (2008).

[25] S. S. Botelho and C. A. R. Sá de Melo, Phys. Rev. Lett. 96, 040404 (2006).

[26] W. Zhang, G.-D. Lin, and L.-M. Duan, Phys. Rev. A 78, 043617 (2008).

[27] R. D. Duncan and C. A. R. Sá de Melo, Phys. Rev. B 62, 9675 (2000).

[28] S. De Palo, C. Castellani, C. Di Castro, and B. K. Chakraverty, Phys. Rev. B 60, 564 (1999).

[29] P. Nozières and S. Schmitt-Rink, J. Low Temp. Phys. 59, 195 (1985); C. A. R. Sá de Melo, M. Randeria, and J. R. Engelbrecht, Phys. Rev. Lett. 71, 3202 (1993).

[30] S. V. Traven, Phys. Rev. Lett. 73, 3451 (1994).

[31] D. S. Petrov, G. V. Shlyapnikov, and J. T. M. Walraven, Phys. Rev. Lett. 85, 3745 (2000).

[32] J. Tempere, M. Wouters, and J. T. Devreese, Phys. Rev. B 75, 184526 (2007).

[33] N. Fukushima, Y. Ohashi, E. Taylor, and A. Griffin, Phys. Rev. A 75, 033609 (2007); E. Taylor, A. Griffin, N. Fukushima, and Y. Ohashi, ibid. 74, 063626 (2006).

[34] D. R. Nelson and J. M. Kosterlitz, Phys. Rev. Lett. 39, 1201 (1977).

[35] D. S. Petrov and G. V. Shlyapnikov, Phys. Rev. A 64, 012706 (2001).

[36] M. M. Parish, F. M. Marchetti, A. Lamacraft, and B. D. Simons, Nat. Phys. 3, 124 (2007).

[37] G. Sarma, J. Phys. Chem. Solids 24, 1029 (1963).

[38] J. Tempere, S. N. Klimin, J. T. Devreese, and V. V. Moshchalkov, Phys. Rev. B 77, 134502 (2008). 